\title{
SLOPE DISTRIBUTION IN FRONT-BACK ASYMMETRIC STOCHASTIC LAGRANGE TIME WAVES
}

\author{
G. LINDGREN, ${ }^{*}$ Lund University
}

\begin{abstract}
The stochastic Lagrange wave model is a realistic alternative to the Gaussian linear wave model, which has been successfully used in ocean engineering for more than half a century. In this paper we present the slope distributions and other characteristic distributions at level crossings for asymmetric Lagrange time waves, i.e. what can be observed at a fixed measuring station, thereby extending results previously given for space waves. The distributions are given as expectations in a multivariate normal distribution, and they have to be evaluated by simulation or numerical integration. Interesting characteristic variables are the slope in time, the slope in space, and the vertical particle velocity when the waves are observed close to instances when the water level crosses a predetermined level. The theory has been made possible by recent generalizations of Rice's formula for the expected number of marked crossings in random fields.
\end{abstract}

Keywords: Crossing theory; Gaussian process; Palm distribution; Rice formula; Slepian model; wave steepness

2010 Mathematics Subject Classification: Primary 60G15

Secondary 60G70; 74J05; 74J30; 76B15; $86 \mathrm{~A} 05$

\section{Introduction}

The stochastic Lagrange wave model is a realistic alternative to the Gaussian linear model, which has been successfully used in ocean engineering for more than half a century. It can produce random waves with the characteristic crest-trough asymmetry of real ocean waves, and its statistical properties can be derived from the power spectrum. Furthermore, it has the physical interpretation that it models the combined vertical and horizontal movements of individual water particles. The nonstochastic Lagrange models were introduced by Gerstner [6], and generalized to finite depths by Miche [13].

Stochastic Lagrange models were introduced and studied by Gjøsund [7], Socquet-Juglard et al. [14], and Fouques et al. [5], who showed that Monte Carlo simulated stochastic Lagrange models can produce realistic crest-trough asymmetry as well as front-back asymmetry, the latter for higher-order Lagrange models. Theoretical studies of their stochastic properties have recently been made by Lindgren [9], Åberg [1], and Åberg and Lindgren [2], covering the basic stochastic properties, slope distributions at level crossings, and height distributions, respectively. These cited works all deal with a first-order stochastic Lagrange model that produces crest-trough statistically asymmetric, but front-back symmetric, random waves, in the sense that the slope distribution at the wave front is the mirror image of that at the rear.

Received 7 January 2009; revision received 8 February 2010.

* Postal address: Mathematical Statistics, Lund University, Box 118, SE-221 00 Lund, Sweden.

Email address: georg@maths.lth.se 
The front-back symmetry is clearly unrealistic, and by introducing a physically motivated correlation between horizontal movement and vertical height, Lindgren and Åberg [11] obtained a stochastic Lagrange model for space waves, i.e. waves observed at a fixed moment in time, with a realistic degree of front-back asymmetry, similar to what has been observed in wave measurements in the ocean or in wave tanks. The slope distributions for the space waves are explicit and depend only on the correlation between the vertical and horizontal processes, and their space derivatives. The distributions in question are the long run observable distribution for slopes observed at level upcrossings or downcrossings in space.

Slope distributions for time waves, i.e. waves recorded at a fixed location in space, are much less explicit than those for space waves, as we show in this paper. By this we mean observations of the wave shapes, including slopes, registered around instances when the time wave crosses a predetermined level. Compared to the space waves, the time waves require a more sophisticated analysis of crossing events, which is made possible by recent generalizations of Rice's formula for the marked number of crossings in random fields, presented in [3, Theorem 6.4].

For engineering purposes, there are many variables that are of interest, related to upcrossings or downcrossings in time. Quantities to be dealt with in this paper are time slopes (TT), space slopes (ST), and horizontal particle speed (VT), in waves observed near level crossings in time. All these quantities are important in the calculation of wave impact, for example, on offshore structures: the TT case shows how fast the water level will rise, once it has reached a high level, the ST case deals with the geometry of the wave when it reaches the high level, and the VT case deals with forces that a wave can exert on a marine or offshore structure. Figure 1 shows examples of front-back asymmetric Lagrange waves in space and time. For details on the model, see Section 5.1.

The free Lagrange model is presented in Section 2, together with two linked models, one physically motivated and one ad hoc, statistical, model. Section 3 contains definitions of
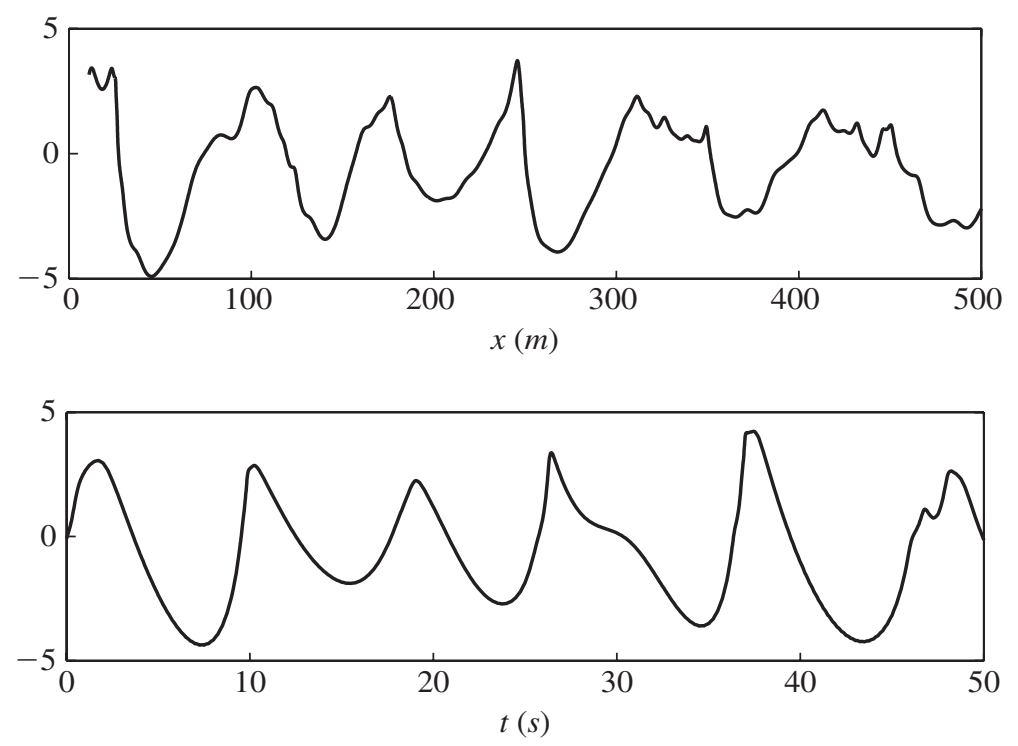

FIGURE 1: Front-back asymmetric Lagrange space wave (top) and time wave (bottom). Note that in the space waves, the downcrossings at the steep (right) wave front correspond to steep upcrossings in the time wave. 
observable distributions and a derivation of the conditional distributions near level crossings; the main result in the paper is Theorem 2. In Section 4, a new measure of front-back asymmetry is suggested, expressed as the asymmetry of the space wave, observed at instances when the time wave has a level crossing. In Section 5 we present some examples which illustrate the basic features of front-back asymmetry. All calculations and simulations were performed in MATLAB ${ }^{\circledR}$ using the WAFO toolbox (see http:www.maths.lth.se/matstat/wafo/). A discussion on the theoretical results in this paper in relation to empirical wave studies is to be found in [10].

The theory presented in this paper works for two-dimensional time waves, i.e. waves with a time coordinate $t$ and a one-dimensional space coordinate $x$ measured along a straight line. The similar theory for three-dimensional waves, i.e. waves with a two-dimensional space parameter, is more complicated, even for space waves, and will be dealt with in a forthcoming paper.

\section{The stochastic Lagrange model}

\subsection{The free stochastic Lagrange model}

The free stochastic Lagrange wave model is a stochastic version of the Miche [13] waves, which, for infinite water depth, reduce to the Gerstner [6] waves. The Gerstner-Miche model describes the vertical and horizontal movements of each water particle as a function of time $t$ and of its original horizontal location, also called the reference coordinate. In the first-order model elementary components with different frequencies and wave numbers act independently of each other, and their effects are added.

2.1.1. The vertical Gaussian process. In the stochastic two-dimensional Gaussian model, the sea surface elevation $W(t, u)$ above the still water level at location $u$ at time $t$ is a Gaussian process with mean 0 . We first consider unidirectional waves where all elementary waves travel from left to right. For a fixed location $u_{0}$, the time-dependent process $W\left(t, u_{0}\right)$ is a stationary Gaussian process with one-sided wave frequency spectrum $S(\omega), \omega>0$. We assume that the spectrum is absolutely continuous, so $W\left(t, u_{0}\right)$ is ergodic, and time averages converge to expected values. The relation between the frequency $\omega$ and the wavenumber $\kappa=\kappa(\omega)$ is given by the depth-dependent dispersion relation

$$
\omega^{2}=g \kappa \tanh \kappa h,
$$

where $g$ denotes the gravitational constant and $h$ is the water depth. We let $\kappa(\omega)$ denote the solution that has the same sign as $\omega$. The covariance function of the time process is then given by

$$
r(t)=\operatorname{cov}\left(W\left(s, u_{0}\right), W\left(s+t, u_{0}\right)\right)=\int_{0}^{\infty} \cos (\omega t) S(\omega) \mathrm{d} \omega=\frac{1}{2} \int_{-\infty}^{\infty} \mathrm{e}^{\mathrm{i} \omega t} S(\omega) \mathrm{d} \omega,
$$

where we have extended the spectrum to negative $\omega$ by setting $S(-\omega)=S(\omega)$, as is customarily done.

The dispersion relation implies the following spectral representation of the corresponding space-time covariance function:

$$
\begin{aligned}
r(t, u) & =\operatorname{cov}(W(s, x), W(s+t, x+u)) \\
& =\int_{0}^{\infty} \cos (\kappa u-\omega t) S(\omega) \mathrm{d} \omega \\
& =\frac{1}{2} \int_{-\infty}^{\infty} \mathrm{e}^{\mathrm{i}(\kappa u-\omega t)} S(\omega) \mathrm{d} \omega .
\end{aligned}
$$


As noted above, in this symmetric form (2) we have defined the wavenumber $\kappa(\omega)$ to satisfy (1) with the same sign as $\omega$.

The spectral representation of the process is

$$
W(t, u)=\int_{-\infty}^{\infty} \mathrm{e}^{\mathrm{i}(\kappa u-\omega t)} \mathrm{d} \zeta(\omega),
$$

where the complex-valued Gaussian process $\zeta(\omega)$ has orthogonal increments such that

$$
\mathrm{E}\left(\mathrm{d} \zeta(\omega) \overline{\mathrm{d} \zeta\left(\omega^{\prime}\right)}\right)= \begin{cases}0 & \text { if } \omega \neq \omega^{\prime} \\ \frac{1}{2} S(\omega) & \text { if } \omega=\omega^{\prime}\end{cases}
$$

Remark 1. In the unidirectional model all waves travel from left to right. Waves in the opposite direction are obtained by letting $\kappa$ and $\omega$ in (3) solve the dispersion relation (1) with different signs. The general bi-directional two-dimensional process is the sum of two independent processes,

$$
W(t, u)=\int_{-\infty}^{\infty} \mathrm{e}^{\mathrm{i}\left(\kappa^{+}(\omega) u-\omega t\right)} \mathrm{d} \zeta^{+}(\omega)+\int_{-\infty}^{\infty} \mathrm{e}^{\mathrm{i}\left(\kappa^{-}(\omega) u-\omega t\right)} \mathrm{d} \zeta^{-}(\omega) .
$$

Here $\kappa^{+}(\omega)$ solves the dispersion relation with the same sign as $\omega$, while $\kappa^{-}(\omega)$ and $\omega$ have opposite signs. The spectrum for $W(t, u)$ need not be symmetric. The main results in Theorem 2, below, are also valid for waves going in two directions.

2.1.2. The free Lagrangian wave process. In the free Lagrangian wave model the horizontal displacement of a water particle with reference coordinate $u$ is obtained as a linear filtration of the vertical process $W(t, u)$ with depth- and frequency-dependent amplitude and phase functions.

The complex form of the filtration of the stochastic process $W(t, u)$ is given by (4), below; see [5]. Thus, the vertical and horizontal components of the free stochastic Lagrangian wave model are defined as two Gaussian processes,

$$
\begin{aligned}
W(t, u) & =\int_{-\infty}^{\infty} \mathrm{e}^{\mathrm{i}(\kappa u-\omega t)} \mathrm{d} \zeta(\omega), \\
X_{M}(t, u) & =u+\int_{-\infty}^{\infty} \mathrm{e}^{\mathrm{i}(\kappa u-\omega t)} \mathrm{i} \frac{\cosh \kappa h}{\sinh \kappa h} \mathrm{~d} \zeta(\omega)=u+\int_{-\infty}^{\infty} \mathrm{e}^{\mathrm{i}(\kappa u-\omega t)} H_{M}(\omega) \mathrm{d} \zeta(\omega) .
\end{aligned}
$$

Here, $\kappa=\kappa(\omega)$ satisfies the dispersion relation (1) with the same sign as $\omega$. The process $X_{M}(t, u)$ is the Miche filtration of $W(t, u)$, with Miche transfer function $H_{M}(\omega)=$ $\mathrm{i} \cosh (\kappa h) / \sinh (\kappa h)$.

The free stochastic Lagrangian wave model is the bivariate Gaussian process

$$
(t, u) \mapsto\left(W(t, u), X_{M}(t, u)\right) ;
$$

a water particle with reference coordinate $u$ has, at time $t$, horizontal location $X_{M}(t, u)$, and the height of the water surface at location $X_{M}(t, u)$ is given by $W(t, u)$. The spectral density $S(\omega)$ for the vertical process $W\left(t, u_{0}\right)$, called the orbital spectrum, differs from the spectrum of the actual water surface process. The free model produces crest-trough asymmetric, but front-back symmetric waves, as described in [1]. 


\subsection{The Lagrange model with linked components}

In the free Lagrange model the individual water particles move according to the simplified hydrodynamic laws of motion, unaffected by outer forces. The dependence between vertical and horizontal movements is taken care of by the Miche filtration. As a consequence, the components $X_{M}$ and $W$ are dependent Gaussian processes, but, for fixed $t$ and $u$, the random variables $X_{M}(t, u)$ and $W(t, u)$ are independent. As it turns out, the statistical front-back symmetry of the free model is coupled to this independence. To obtain the necessary asymmetry, the model has to exhibit a special correlation between the vertical and horizontal processes and their derivatives.

2.2.1. A physically based linked model. For wind driven waves, the interaction between wind and waves is complex, and a stochastic description seems out of reach, at least at present. To keep a stochastic model within the Gaussian realm, with reasonable physical motivation, we will here introduce additional terms besides the Miche filtration to let the vertical height process affect the horizontal movements in a physically realistic way. By taking the additional term as a linear filtration of the vertical process, the two processes are still jointly Gaussian, so the theory developed in [1] can be applied.

A physically motivated relation between the vertical and horizontal processes is obtained by letting the horizontal acceleration of the water particles depend linearly on the vertical height and slope, for example to take $X(t, u)$ as the solution to an equation

$$
\frac{\partial^{2} X(t, u)}{\partial t^{2}}=\frac{\partial^{2} X_{M}(t, u)}{\partial t^{2}}-\alpha W(t, u)
$$

where $\alpha>0$.

With $G(\omega)=-\alpha /(-\mathrm{i} \omega)^{2}$ and the transfer function

$$
H(\omega)=H_{M}(\omega)+G(\omega)=\mathrm{i} \frac{\cosh \kappa h}{\sinh \kappa h}-\frac{\alpha}{(-\mathrm{i} \omega)^{2}}=\rho(\omega) \mathrm{e}^{\mathrm{i} \theta(\omega)}, \quad \text { say, }
$$

the horizontal movement process for unidirectional waves is expressed in the standard form (with $\kappa$ and $\omega$ of the same sign),

$$
X(t, u)=u+\int_{-\infty}^{\infty} \mathrm{e}^{\mathrm{i}(\kappa u-\omega t+\theta(\omega))} \rho(\omega) \mathrm{d} \zeta(\omega) .
$$

Comparing (7) with (4), we see that the free Lagrange model represents a phase shift between vertical and horizontal movements of $\theta=\pi / 2=90$ degrees, while the general model has a frequency-dependent phase shift.

2.2.2. A forced correlation model. The distributions of slopes in the linked Lagrange process will depend only on the correlations between the vertical and horizontal processes, and their time and space derivatives. It is unimportant for the theory how these correlations have been generated. In the ideal situation, we should base the correlation structure on authentic measurements of water particle movements.

In the physically motivated model (5), the vertical elevation influences the horizontal movements in an indirect way. As an alternative, we could define a direct dependence relation between the vertical and horizontal processes, by taking, for example,

$$
X(t, u)=\gamma X_{M}(t, u)+\delta W(t, u),
$$

with constants $\gamma$ and $\delta$ corresponding to the transfer function $H(\omega)=\gamma H_{M}(\omega)+\delta$. 
For practical use of the theory, we only have to specify a few correlations. The special models (6) and (8) will be seen as reasonable examples of how the correlations may appear. In the examples in Section 5 we will see some of the differences between the two models.

\subsection{Time and space waves}

The full stochastic two-dimensional Lagrangian model is a bivariate Gaussian process of a time and space parameter $(t, u)$. It is then important to discriminate between the observable time and space wave formulations.

The space wave is obtained as the parametric curve

$$
u \mapsto\left(X\left(t_{0}, u\right), W\left(t_{0}, u\right)\right),
$$

by keeping time $t=t_{0}$ fixed. It resembles the free water surface profile $L\left(t_{0}, x\right)$ along the studied direction. A complication in the model is that there may well occur double points, where $X\left(t_{0}, u_{1}\right)=X\left(t_{0}, u_{2}\right)$ with $u_{1} \neq u_{2}$ and $W\left(t_{0}, u_{1}\right) \neq W\left(t_{0}, u_{2}\right)$. The space wave is defined implicitly through the relation

$$
L\left(t_{0}, X\left(t_{0}, u\right)\right)=W\left(t_{0}, u\right)
$$

and explicitly, if there is only one $u=X^{-1}\left(t_{0}, x\right)$ satisfying $X\left(t_{0}, u\right)=x$, as

$$
L\left(t_{0}, x\right)=W\left(t_{0}, X^{-1}\left(t_{0}, x\right)\right) .
$$

Until recently, there has been few observational, detailed studies on the space process, except for photos and low resolution satellite reconstructions, which do not permit us to draw conclusions about elevation/velocity relations. The time wave is more often registered and reported. It is obtained as the time series of measurements of the free water level $L\left(t, x_{0}\right)$ at a fixed location in space with coordinate $x_{0}$, viz. the curve

$$
t \mapsto W\left(t, X^{-1}\left(t, x_{0}\right)\right)
$$

provided that the inverse $X^{-1}\left(t, x_{0}\right)=\left\{u ; X(t, u)=x_{0}\right\}$ is uniquely defined at time $t$. Then, there is only one water particle located at position $x_{0}$ at that time. Otherwise, the Lagrangian time wave takes multiple values.

Figure 1 shows typical examples of Lagrange space and time waves for shallow water, $h=8 \mathrm{~m}$, and strong coupling, $\alpha=0.8$ in model (6). Observe that the space waves, moving from left to right, have a steep right front and a less steep left back, which corresponds to the reversed skewness in the time wave, when plotted as a function of time.

The time and space partial derivatives

$$
L_{t}(t, x)=\frac{\partial L(t, x)}{\partial t} \quad \text { and } \quad L_{x}(t, x)=\frac{\partial L(t, x)}{\partial x}
$$

will be defined and studied in detail in the next section (see (9) and (10), below).

\section{Crossing distribution for time waves}

\subsection{Crossing related wave characteristics}

The Lagrange time wave is the sea level variation measured at a fixed location. The statistical distribution of wave characteristics has to be interpreted in a frequentistic way as what we can 
empirically observe in an infinitely extended, statistically stationary recording of the sea level. In different applications we can identify many different characteristic quantities related to the wave profiles. Some of them are just described by their statistical distribution when the waves are sampled at a constant sampling rate. Others are coupled only to level crossings in time and the wave profile near time instances when the wave reaches some specified level above or below the mean water level. Some variables of interest for the time wave formulation are as follows.

(TT) Slope in time at level crossings in time. This is the distribution of the slope $L_{t}\left(t, x_{0}\right)$ observed only at the upcrossings or downcrossings of a fixed level $v$ by the time wave $L\left(t, x_{0}\right)$ (synchronous sampling). Note that this is the rate of increase of the water level at the times when it has already reached level $v$.

(ST) Slope in space at level crossings in time. This is the distribution of the space slope $L_{x}\left(t, x_{0}\right)$ observed at the instances when the time wave reaches level $v$. Note that this is the slope of the moving wave front that may hit an upper deck of an offshore construction when a high wave reaches the deck.

(VT) Profile velocity at level crossings in time. This is the distribution of the horizontal velocity $X_{t}\left(t, x_{0}\right)$ of the water particles at the times when the wave reaches level $v$. Note that this is a quantity of interest for the impact on a structure in case of an extreme wave.

Åberg [1] derived expressions for the statistical distribution of the slope at crossings of any fixed level, for the (TT) case for time slopes in time waves and the analogous (SS) case, slopes in space for the space waves, but only for the free Lagrange model with statistically front-back symmetric waves. Lindgren and Åberg [11] have given the modification required to get the explicit slope distribution (SS) at upcrossings and downcrossings for the asymmetric space waves in the linked Lagrange model.

For space waves, the analysis is based on the fact that the slopes at upcrossings or downcrossings in a Gaussian stationary process have a simple Rayleigh distribution. For the time waves, the situation is more complex, since we need to keep track of what particle is presently located at the measure station. Therefore, the relevant conditional slope distributions will have a much more complicated distribution; see [10].

In the following we will derive expressions for the three variables described above, but first we define how to interpret conditional distributions in this context.

\subsection{Observable distributions}

3.2.1. Some definitions. Before we derive the distributions of slopes and other related variables, we need to define precisely what is meant by an observable distribution in the time wave. A statistical distribution is always related to an empirical experiment, and for the time wave at location $x_{0}$, we consider a continuous series of observations of the process $L\left(t, x_{0}\right), t \geq 0$, with multiplicity in case it is not unique. We will derive the observable distributions in terms of the partial time and space derivatives of the processes $X(t, u)$ and $W(t, u)$, which we denote as

$$
\begin{aligned}
X_{t}(t, u) & =\frac{\partial X(t, u)}{\partial t}, & X_{u}(t, u) & =\frac{\partial X(t, u)}{\partial u}, \\
W_{t}(t, u) & =\frac{\partial W(t, u)}{\partial t}, & W_{u}(t, u) & =\frac{\partial W(t, u)}{\partial u},
\end{aligned}
$$

etc. 
The Lagrange time waves satisfy, by definition, the relation $L(t, X(t, u))=W(t, u)$. By differentiating with respect to $t$, we obtain

$$
\frac{\partial L(t, X(t, u))}{\partial t}=W_{t}(t, u)=L_{t}(t, X(t, u))+L_{u}(t, X(t, u)) X_{t}(t, u) .
$$

By differentiating with respect to $u$, we further obtain

$$
W_{u}(t, u)=L_{u}(t, X(t, u)) X_{u}(t, u),
$$

giving the fundamental definition of the time wave slope at location $X(t, u)$,

$$
L_{t}(t, X(t, u))=W_{t}(t, u)-W_{u}(t, u) \frac{X_{t}(t, u)}{X_{u}(t, u)} .
$$

Equation (9) is a mathematical identity, and if $X^{-1}\left(t, x_{0}\right)$ is uniquely defined, it also gives the unique slope of the Lagrange time wave $L\left(t, x_{0}\right)$ at location $x_{0}$. If there are multiple $u$-values such that $X(t, u)=x_{0}$, then we define $L_{t}\left(t, x_{0}\right)$ by (9) for each of these $u$-values.

We also need the slope of the space wave observed at a time $t_{0}$, which is either fixed, or random and determined by the wave process itself. The space wave is implicitly defined by $L\left(t_{0}, X\left(t_{0}, u\right)\right)=W\left(t_{0}, u\right)$, or explicitly, if there is only one $u=X^{-1}\left(t_{0}, x\right)$ satisfying $X\left(t_{0}, u\right)=x$, by $L\left(t_{0}, x\right)=W\left(t_{0}, X^{-1}\left(t_{0}, x\right)\right)$. Its slope is defined by

$$
L_{x}\left(t_{0}, x\right)=\frac{W_{u}\left(t_{0}, u\right)}{X_{u}\left(t_{0}, u\right)},
$$

with the understanding that (9) defines the slope for each solution, if there are many.

To simplify the notation, we also introduce the vectors

$$
\begin{aligned}
& \boldsymbol{Z}(t, u)=(W(t, u), X(t, u))^{\top}, \\
& \boldsymbol{Y}(t, u)=\left(W_{t}(t, u), W_{u}(t, u), X_{t}(t, u), X_{u}(t, u)\right)^{\top},
\end{aligned}
$$

for the basic processes and their partial derivatives.

3.2.2. Distributions conditioned on level crossings. To deal with cases (TT), (ST), and (VT), we need to find, for the fixed location $x_{0}$, the observable distributions of $L_{t}(t, X(t, u))$, $L_{u}(t, X(t, u))$, and $X_{t}(t, u)$, over all instances when $W(t, u)=v$ and $X(t, u)=x_{0}$. This means that we have to identify all combinations of time $t$ and reference points $u$ at which, simultaneously, $W(t, u)=v$ and $X(t, u)=x_{0}$. Thus, we have to deal with a crossing problem for two dependent Gaussian fields.

Figure 2 illustrates the idea of time wave crossings of the level $v=2$, at location $x_{0}=0$. It shows simultaneous level curves for the $X(t, u)$-field and the $W(t, u)$-field. The thick solid curve is the set where $X(t, u)=0$, which means that the time wave is defined as the values of $W(t, u)$ along this curve. Note that in this exaggerated example there are multiple points, so that, e.g. there are three $u$-values for which $X(4, u)=0$. The $W(t, u)$-field is equal to $v=2$ along the dotted lines and equal to $v=0$ along the dashed lines. Obviously, in the time wave $L(t, 0)$ there is an upcrossing of level $v=2$ near time $t=4$, and a downcrossing of the same level very close, but not exactly equal, in time.

Assume that the observation interval is $[0, T]$, and let $t_{k} \in[0, T]$ be the times when $L\left(t, x_{0}\right)=v$, i.e. there exists at least one particle with reference coordinate $u$ such that 


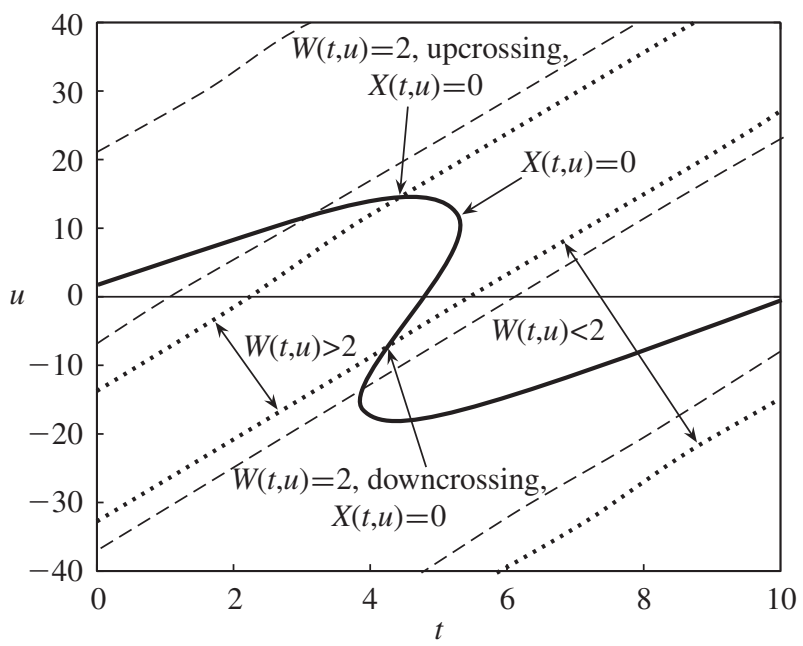

Figure 2: Crossings event in time wave defined by crossing of the level curves for $W(t, u)$ and those for $X(t, u)$. Particles with reference coordinates on the thick solid curve will, at time $t$, be located at $x_{0}=0$. The height field $W(t, u)$ is equal to $v=2$ along the dotted lines, and equal to $v=0$ along the dashed lines. Thus, at the event marked 'upcrossing', the time wave at location $x_{0}=0$ goes from being less than $v=2$ to being greater than $v=2$.

$X\left(t_{k}, u\right)=x_{0}$ and $W\left(t_{k}, u\right)=v$. It will follow from the generalized Rice formula (13), below, that there is, with probability 1 , only one such particle, which we can denote by $u_{k}$, so that $X\left(t_{k}, u_{k}\right)=x_{0}$. In analogy with (11) and (12), we write $z_{k}=\left(v, x_{0}\right)^{\top}$ and $\boldsymbol{y}_{k}=\boldsymbol{Y}\left(t_{k}, u_{k}\right)$ for the observed values at the crossings.

To find the statistical distributions for the quantities in cases (TT), (ST), and (VT), we need the conditional distribution of the reference coordinate and the relevant derivatives given the crossing event at the times $t_{k}$.

\subsection{Conditional distributions at level crossings in time waves}

3.3.1. Crossing and marked crossings intensity. The interesting wave characteristics defined by, e.g. (9) and (10), contain the partial derivatives at a random reference coordinate, and, thus, we have to find their observable simultaneous distribution, conditioned on the crossing event. To this end, we need a generalized form of Rice's formula for the intensity of marked crossings in multiple Gaussian fields; see [3, Theorem 6.4] and [12]. Define

$$
N_{T}=\#\left\{(t, u) \in[0, T] \times \mathbb{R} ; W(t, u)=v, X(t, u)=x_{0}\right\}=\#\left\{t_{k} \in[0, T]\right\},
$$

and write, for any Borel set $A \subset \mathbb{R}^{5}$,

$$
\begin{aligned}
N_{T}(A) & =\#\left\{(t, u) \in[0, T] \times \mathbb{R} ; W(t, u)=v, X(t, u)=x_{0},\left(u, \boldsymbol{Y}(t, u)^{\top}\right)^{\top} \in A\right\} \\
& =\#\left\{t_{k} \in[0, T] ;\left(u_{k}, \boldsymbol{y}_{k}^{\top}\right)^{\top} \in A\right\}
\end{aligned}
$$

for the observed total number, in the time interval $[0, T]$, of time wave crossings of the level $v$, and the number of such crossings with the specified restriction on the reference coordinate and derivatives, respectively. 
The vector $\left(u_{k}, \boldsymbol{y}_{k}^{\top}\right)^{\top}$ acts as a mark attached to the crossing at $t_{k}$ and the ratio $N_{T}(A) / N_{T}$ defines the empirical distribution of the observed marks. As remarked in Section 2.1.1, for an ergodic process, the ratio converges as $T \rightarrow \infty$ to the ratio $\mathrm{E}\left(N_{1}(A)\right) / \mathrm{E}\left(N_{1}\right)$ between the intensity of marked and unmarked crossings, which therefore defines the observable distribution of marks at time wave crossings.

Let $f_{W(0, u), X(0, u)}\left(v, x_{0}\right)$ be the joint Gaussian density function of $W(0, u)$ and $X(0, u)$, and write $p(\boldsymbol{y} ; u)$ for the conditional Gaussian density of $\boldsymbol{Y}(t, u)$ given that $\boldsymbol{Z}(t, u)=(v, 0)^{\top}$, evaluated at $\boldsymbol{y}=\left(w_{t}, w_{u}, x_{t}, x_{u}\right)^{\top}$. (Stationarity in time implies that the density does not depend on $t$.) It then follows from Lemma 2 of [12] (see also [1]) that

$$
\mathrm{E}\left(N_{1}(A)\right)=\int_{-\infty}^{\infty} g_{A}(u) f_{W(0, u), X(0, u)}\left(v, x_{0}\right) \mathrm{d} u,
$$

where

$$
\begin{aligned}
g_{A}(u)= & \mathrm{E}\left(\left|W_{t}(0, u) X_{u}(0, u)-W_{u}(0, u) X_{t}(0, u)\right|\right. \\
& \left.\times \mathbf{1}\left(\left(u, \boldsymbol{Y}(0, u)^{\top}\right)^{\top} \in A\right) \mid W(0, u)=v, X(0, u)=x_{0}\right) \\
= & \iiint \int_{\left(u, \boldsymbol{y}^{\top}\right)^{\top} \in A}\left|w_{t} x_{u}-w_{u} x_{t}\right| p(\boldsymbol{y}, u) \mathrm{d} \boldsymbol{y},
\end{aligned}
$$

where $1(A)$ denotes the indicator function of the event $A$.

Furthermore, we get, from the same lemma,

$$
\mathrm{E}\left(N_{1}\right)=\int_{-\infty}^{\infty} g(u) f_{W(0, u), X(0, u)}(v, 0) \mathrm{d} u,
$$

with

$$
\begin{aligned}
g(u) & =\mathrm{E}\left(\left|W_{t}(0, u) X_{u}(0, u)-W_{u}(0, u) X_{t}(0, u)\right| \mid W(0, u)=v, X(0, u)=x_{0}\right) \\
& =\iiint \int_{\left(u, \boldsymbol{y}^{\top}\right)^{\top} \in \mathbb{R}^{5}}\left|w_{t} x_{u}-w_{u} x_{t}\right| p(\boldsymbol{y}, u) \mathrm{d} \boldsymbol{y} .
\end{aligned}
$$

By choosing appropriate sets $A$ we will later be able to give satisfying solutions to problems (TT)-(VT). Note that the integrals (13) and (14) are independent of the location $x_{0}$, at which the time wave is observed.

3.3.2. Explicit expressions. To get explicit expressions for the densities, we refer to the fact that the joint distribution of $\boldsymbol{Y}(t, u)$ and $\boldsymbol{Z}(t, u)$ is six-dimensional Gaussian, with mean $\boldsymbol{\mu}$ and covariance matrix $\boldsymbol{\Sigma}$, partitioned according to (23) and (24) in Appendix A.

Furthermore, from (25) and (26) in Appendix A, the conditional distribution of $\boldsymbol{Y}(t, u)$ given $\boldsymbol{Z}(t, u)=\left(v, x_{0}\right)^{\top}$ is four-dimensional Gaussian with mean and covariance matrix independent of $t$, and given by

$$
\begin{gathered}
\boldsymbol{\mu}_{\boldsymbol{Y} \mid \boldsymbol{Z}=\left(v, x_{0}\right)^{\top}}=\boldsymbol{A}+v \boldsymbol{B}+\left(u-x_{0}\right) \boldsymbol{C}, \\
\boldsymbol{\Sigma}_{\boldsymbol{Y} \mid \boldsymbol{Z}}=\boldsymbol{\Sigma}_{\boldsymbol{Y} \boldsymbol{Y}}-\boldsymbol{\Sigma}_{\boldsymbol{Y} \boldsymbol{Z}} \boldsymbol{\Sigma}_{\boldsymbol{Z} \boldsymbol{Z}}^{-1} \boldsymbol{\Sigma}_{\boldsymbol{Z} \boldsymbol{Y}}
\end{gathered}
$$

The following theorem about the long-run observable distribution then follows directly from (13). 
Theorem 1. The long-run empirical distribution of reference coordinates $u_{k}$ and partial derivatives $\boldsymbol{y}_{k}$ at the $v$-level crossings $t_{k}$, of the Lagrange time wave, has density function

$$
\begin{aligned}
f\left(u, w_{t}, w_{u}, x_{t}, x_{u}\right)= & \frac{1}{\mathrm{E}\left(N_{1}\right)}\left|w_{t} x_{u}-w_{u} x_{t}\right| p\left(w_{t}, w_{u}, x_{t}, x_{u} ; u\right) \\
& \times \frac{1}{2 \pi \sqrt{\operatorname{det} \Sigma_{Z Z}}} \exp \left\{-\frac{1}{2}(v,-u) \Sigma_{Z Z}^{-1}(v,-u)^{\top}\right\} .
\end{aligned}
$$

Proof. The empirical distribution of $\left(u_{k}, \boldsymbol{y}_{k}^{\top}\right)^{\top}$ for $t_{k} \in[0, T]$ converges, as the observation interval increases,

$$
\frac{N_{T}(A)}{N_{T}} \rightarrow \frac{\mathrm{E}\left(N_{1}(A)\right)}{\mathrm{E}\left(N_{1}\right)} \quad \text { as } T \rightarrow \infty,
$$

which gives the stated density.

Remark 2. In the free Lagrange model [1], the $w$-variables and the $x$-variables are conditionally independent, given $\boldsymbol{Z}=\left(v, x_{0}\right)^{\top}$, which considerably simplifies the analysis.

Remark 3. The distribution (15) can be used to construct a Slepian model for the conditional fields around the crossings in the Lagrange time wave. The Slepian model will consist of one regression term on a vector $\left(U, \boldsymbol{Y}^{\top}\right)^{\top}$ with density (15), and one Gaussian residual term. By analyzing these conditional fields we can get detailed information of the exact wave forms in the Lagrange time model. Lindgren [9] derived a similar Slepian model conditioned on a different crossing event, namely a crossing in the vertical process $W\left(t, x_{0}\right)$. These crossings do not appear exactly at the same instances as the simultaneous crossings $t_{k}$, and the two Slepian models are therefore different. The one in [9] has a much simpler structure, with only the Rayleigh-distributed slope at the $W\left(t, u_{0}\right)$-crossing in the regression term.

The full Slepian model makes it possible to derive the long-run empirical distributions of all wave characteristics, coupled to the level crossings in the time wave. We will not use the Slepian model explicitly in this work, but rather, in the next section, give the distribution of the specified wave characteristics at the level crossings.

\subsection{Specific distributions at level crossings}

The distribution in Theorem 1 gives the long-run observable distribution of the reference coordinate and partial derivatives, observed at time wave crossings of the level $v$. Since the quantities in problems (TT)-(VT) are all defined in terms of these variables, we can find the wanted distributions by specifying certain sets $A$, and taking the expectation of the corresponding indicator function. Take, for the three cases, the time and space slopes, (9) and (10), and the horizontal particle velocity, and define

$$
\begin{aligned}
&(\mathrm{TT}): g_{y}^{(\mathrm{TT})}(u)=\mathrm{E}(\left|W_{t}(0, u) X_{u}(0, u)-W_{u}(0, u) X_{t}(0, u)\right| \\
& \times \mathbf{1}\left(W_{t}(0, u)-W_{u}(0, u) \frac{X_{t}(0, u)}{X_{u}(0, u)} \leq y\right) \mid W(0, u)=v \\
&\left.X(0, u)=x_{0}\right),
\end{aligned}
$$




$$
\begin{aligned}
(\mathrm{ST}): g_{y}^{(\mathrm{ST})}(u)=\mathrm{E}( & \left|W_{t}(0, u) X_{u}(0, u)-W_{u}(0, u) X_{t}(0, u)\right| \\
\quad & \left.\times \mathbf{1}\left(\frac{W_{u}(0, u)}{X_{u}(0, u)} \leq y\right) \mid W(0, u)=v, X(0, u)=x_{0}\right), \\
(\mathrm{VT}): g_{y}^{(\mathrm{VT})}(u)=\mathrm{E}( & \left|W_{t}(0, u) X_{u}(0, u)-W_{u}(0, u) X_{t}(0, u)\right| \\
& \left.\times \mathbf{1}\left(X_{t}(0, u) \leq y\right) \mid W(0, u)=v, X(0, u)=x_{0}\right) .
\end{aligned}
$$

Since our goal is to illustrate the front-back asymmetry of the coupled model, we have to separate the crossings into upcrossings and downcrossings, by the extra requirement that the slopes are positive or negative, respectively. This is achieved by including the extra requirement into the indicator function that the time slope,

$$
W_{t}(0, u)-W_{u}(0, u) \frac{X_{t}(0, u)}{X_{u}(0, u)},
$$

is positive or negative, respectively. Write $N^{+}$and $N^{-}$, in analogy with $N$, for the number of upcrossings and downcrossings, respectively, of the level $v$. We then have the following theorem for the distributions at level crossings in the Lagrange time waves for the three cases.

Theorem 2. The cumulative long-run observable distribution function for time slopes at upcrossings of the level $v$ in the Lagrange time wave, observed at location $x_{0}$, is given by

$$
F_{v}^{\mathrm{TT}+}(y)=\frac{1}{\mathrm{E}\left(N^{+}\right)} \int_{-\infty}^{\infty} g_{y}^{\mathrm{TT}+}(u) f_{W(0, u), X(0, u)}\left(v, x_{0}\right) \mathrm{d} u,
$$

where

$$
\begin{aligned}
g_{y}^{\mathrm{TT}+}(u)=\mathrm{E}( & \left|W_{t}(0, u) X_{u}(0, u)-W_{u}(0, u) X_{t}(0, u)\right| \\
& \quad \times \mathbf{1}\left(0<W_{t}(0, u)-W_{u}(0, u) \frac{X_{t}(0, u)}{X_{u}(0, u)} \leq y\right) \mid W(0, u)=v, \\
\mathrm{E}\left(N^{+}\right)=\int_{-\infty}^{\infty} g_{\infty}^{\mathrm{TT}+}(u) f_{W(0, u), X(0, u)}\left(v, x_{0}\right) \mathrm{d} u . & \left.X(0, u)=x_{0}\right),
\end{aligned}
$$

The cumulative distribution function of slopes at downcrossings is obtained by replacing the interval $(0, y]$ by the interval $[-y, 0)$.

The distributions for the space slope and velocity, (ST) and (VT), are found by obvious modifications of (17) and (18).

The conditional expectations in (20) and (21) contain absolute values of products and ratios of normal variables. In this paper, they are found by simulation of the four-dimensional conditional normal variables, followed by numerical integration. An alternative could be to use the RINDmodule in the WAFO toolbox (see http://www.maths.lth.se/matstat/wafo/ and also [8]).

\section{Measures of asymmetry}

To compare the theoretical slope distributions from Theorem 2 with empirically obtained data, we need to summarize the distributions in a front-back asymmetry measure. In the subsequent examples we will illustrate the wave asymmetry by means of the cumulative 


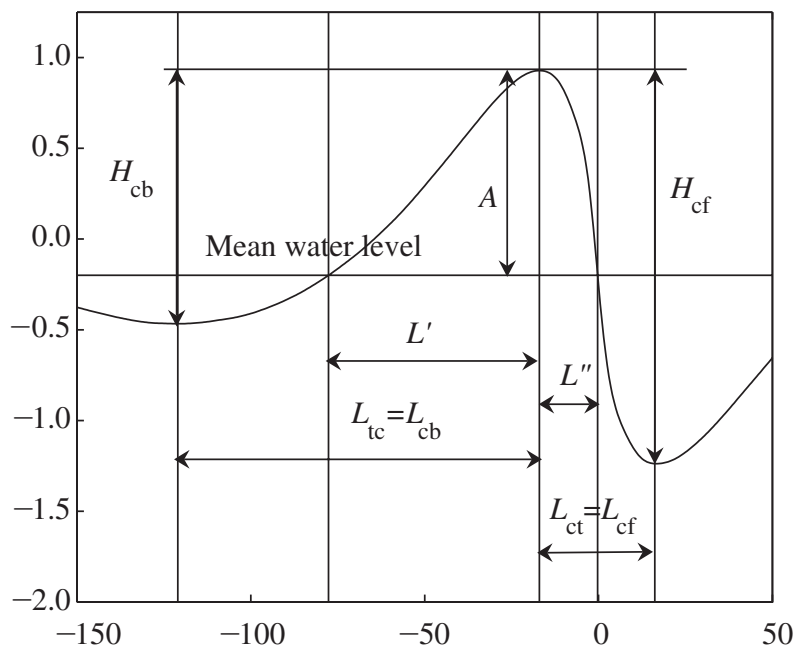

FIGURE 3: Definition of asymmetry parameters; space wave moving from left to right. The subscripts cb and cf refer to crest-back and crest-front definitions, and the subscripts tc and ct refer to trough-crest and crest-trough definitions.

distribution of the time and space wave slopes at upcrossings and downcrossings of specified levels. Figure 3 illustrates the definitions on a typical asymmetric wave profile in space, moving in time from left to right. We should then remember that the corresponding time wave, measured at a fixed point, for example, at $x_{0}=0$, will have a reversed asymmetry, with a fast raising level followed by a gentle decrease. Plotted as a function of time, it would have the steep sides facing left. For the time wave, we denote the characteristic quantities by the letter $T$ instead of $L$.

We will use a definition of asymmetry based on the wave slopes at the upcrossings and downcrossings, $t_{\mathrm{up}}$ and $t_{\mathrm{do}}$, of the mean water level, at either side of the wave crest. With $L_{t}(t)$ denoting the derivative of the time wave $L\left(t, x_{0}\right)$, the asymmetry measure we will use is the ratio

$$
\lambda_{\mathrm{AL}}=-\frac{L_{t}\left(t_{\mathrm{do}}\right)}{L_{t}\left(t_{\mathrm{up}}\right)} \approx \frac{H_{\mathrm{cf}} / T_{\mathrm{cf}}}{H_{\mathrm{cb}} / T_{\mathrm{cb}}} .
$$

Another measure of asymmetry of a signal is the skewness of its Hilbert transform; see [4]. If $L(t)=L\left(t, x_{0}\right)$ is the Lagrange time wave and $\widehat{L}(t)$ is its Hilbert transform with standard deviation $\sigma$, then

$$
A_{\mathrm{H}}=\mathrm{E}\left(\widehat{L}(t)^{3}\right) / \sigma^{3}
$$

is related to the front-back asymmetry of the signal. The time signal corresponding to a space wave such as that in Figure 3, with a gentle rear (left side) and steep drop (right side) and peeks tilted forward in space, will have a negative $A$-value.

We will now introduce a new asymmetry measure based on combined space-time properties. In Section 3.4, we derived the distribution of different wave characteristics observed at the instances when the time wave reaches a specified level, from below at an upcrossing, or from above at a downcrossing. In particular, case (ST) gives the shape of the space wave at these instances. Let $m_{v}^{\mathrm{ST}+}$ be the median of the space slope distribution observed at time upcrossings of the level $v$, i.e. $F_{v}^{\mathrm{ST}+}\left(m_{v}^{\mathrm{ST}+}\right)=0.5$, and let $m_{v}^{\mathrm{ST}-}$ be the median in the space slope distribution at time wave downcrossings of the same level. In the examples we will describe the space-time 
asymmetry by $\lambda_{\mathrm{ST}}=-m_{v}^{\mathrm{ST}+} / m_{v}^{\mathrm{ST}-}$, where $v$ can be given different values according to the application. Note that this definition differs from (22), which relates to the time wave slopes at the time wave crossings, while $\lambda_{\mathrm{ST}}$ relates to the space wave slopes at time wave crossings.

The examples of front-back asymmetry in the following section are based on one orbital spectrum. A discussion of the relations to real wave data and observed asymmetry measures can be found in [10].

\section{Examples}

The front-back asymmetry of the proposed model can be seen both in the distributions conditioned on upcrossings and downcrossings, respectively, of selected fixed levels, and in the asymmetry of an average wave. We will illustrate this for a model with a Pierson-Moskowitz (PM) orbital spectrum, with spectral density

$$
S(\omega)=\frac{5 H_{\mathrm{s}}^{2}}{\omega_{\mathrm{p}}\left(\omega / \omega_{\mathrm{p}}\right)^{5}} \mathrm{e}^{-5\left(\omega / \omega_{\mathrm{p}}\right)^{-4} / 4}, \quad 0<\omega<\omega_{\mathrm{c}}
$$

where $H_{\mathrm{s}}=4 \sqrt{\operatorname{var}(W(t, u))}$ is the significant wave height, and $\omega_{\mathrm{p}}$ is the peak frequency, at which the spectral density has its maximum. The peak period is defined as $T_{\mathrm{p}}=2 \pi / \omega_{\mathrm{p}}$. We use a fixed value $H_{\mathrm{s}}=7 \mathrm{~m}$ for the significant wave height, and assume a finite cut off frequency $\omega_{\mathrm{c}}$ to obtain finite spectral moments and avoid small but high frequency wave components. The steepness parameter, $S=H_{\mathrm{s}} / T_{\mathrm{p}}^{2}$, is important for the degree of front-back asymmetry, and to see the effects, we illustrate the distributions for steep waves with $T_{\mathrm{p}}=12 \mathrm{~s}$. The cut off frequency is $\omega_{\mathrm{c}}=32 / T_{\mathrm{p}}$.

\subsection{The linked model}

We do the calculations for three different depths, $h=8,32, \infty \mathrm{m}$ and with three different degrees of linkage, $\alpha=0,0.4,0.8$, in the linked model (5), where $\alpha=0$, means 'no linkage'.

Table 1 gives some asymmetry measures for the nine cases: the Hilbert transform skewness measure, $A$, the $\lambda_{\mathrm{AL}}$ measure, and the new measure, $\lambda_{\mathrm{ST}}$, based on the space slopes at time crossings of a level one standard deviation above the still water level. As can be seen, the front-back skewness measure is not so much dependent on the water depth as on the degree

TABLE 1: Asymmetry measures for the linked model using the PM spectrum with $H_{\mathrm{s}}=7$ and $T_{\mathrm{p}}=11$.

\begin{tabular}{cccc}
\hline & \multicolumn{3}{c}{$\alpha$} \\
\cline { 2 - 4 } & 0.0 & 0.4 & 0.8 \\
\hline$\infty$ & $A_{\mathrm{H}}=0$ & $A_{\mathrm{H}}=-0.10(-0.18)$ & $A_{\mathrm{H}}=-0.21(-0.34)$ \\
& $\lambda_{\mathrm{AL}}=1$ & $\lambda_{\mathrm{AL}}=1.30(1.55)$ & $\lambda_{\mathrm{AL}}=1.83(3.01)$ \\
& $\lambda_{\mathrm{ST}}=1$ & $\lambda_{\mathrm{ST}}=1.24(1.44)$ & $\lambda_{\mathrm{ST}}=1.52(2.09)$ \\
32 & $A_{\mathrm{H}}=0$ & $A_{\mathrm{H}}=-0.12(-0.2)$ & $A_{\mathrm{H}}=-0.26(-0.43)$ \\
& $\lambda_{\mathrm{AL}}=1$ & $\lambda_{\mathrm{AL}}=1.34(1.60)$ & $\lambda_{\mathrm{AL}}=1.98(3.32)$ \\
& $\lambda_{\mathrm{ST}}=1$ & $\lambda_{\mathrm{ST}}=1.27(1.47)$ & $\lambda_{\mathrm{ST}}=1.59(2.18)$ \\
8 & $A_{\mathrm{H}}=0$ & $A_{\mathrm{H}}=-0.19(-0.35)$ & $A_{\mathrm{H}}=-0.44(-0.65)$ \\
& $\lambda_{\mathrm{AL}}=1$ & $\lambda_{\mathrm{AL}}=1.52(1.98)$ & $\lambda_{\mathrm{AL}}=3.11(4.07)$ \\
& $\lambda_{\mathrm{ST}}=1$ & $\lambda_{\mathrm{ST}}=1.48(1.77)$ & $\lambda_{\mathrm{ST}}=2.13(2.76)$ \\
\hline
\end{tabular}


of linkage. This can also be seen in the slope distribution functions in the next sections. (In Table 1 , the numbers in parentheses are the asymmetry measures in the forced model, to be described in Section 5.2.)

5.1.1. Slope in time at time crossings. The distribution of the time slope

$$
W_{t}\left(t_{k}, u_{k}\right)-W_{u}\left(t_{k}, u_{k}\right) \frac{X_{t}\left(t_{k}, u_{k}\right)}{X_{u}\left(t_{k}, u_{k}\right)}
$$

at upcrossing or downcrossing times $t_{k}$ of a fixed level $v$ is given by (19) in Theorem 2; case (TT). It represents the speed by which the water surface rises when it has reached level $v$. The distribution is found by simulation of the conditional expectation in (20).

Figure 4 illustrates the asymmetry of the average wave at upcrossing and downcrossings of the mean water level. The orbital spectrum is the PM spectrum with $H_{\mathrm{s}}=7 \mathrm{~m}$ and $T_{\mathrm{p}}=12 \mathrm{~s}$. The solid curves give the average time wave shape centered at the time of upcrossing and the dashed curves give the (reversed) average wave centered at downcrossings. The mean water level is negative, due to the crest-trough asymmetry.

Another means to describe the asymmetry is by the distribution functions for the slope at crossings of specified level. Figure 5 shows the time slope distributions at time wave crossings. For $\alpha=0$, the upcrossing and downcrossing distributions are equal, but, for $\alpha \neq 0$, there is a considerable asymmetry at shallow water. There is then also a large probability of very high slopes.
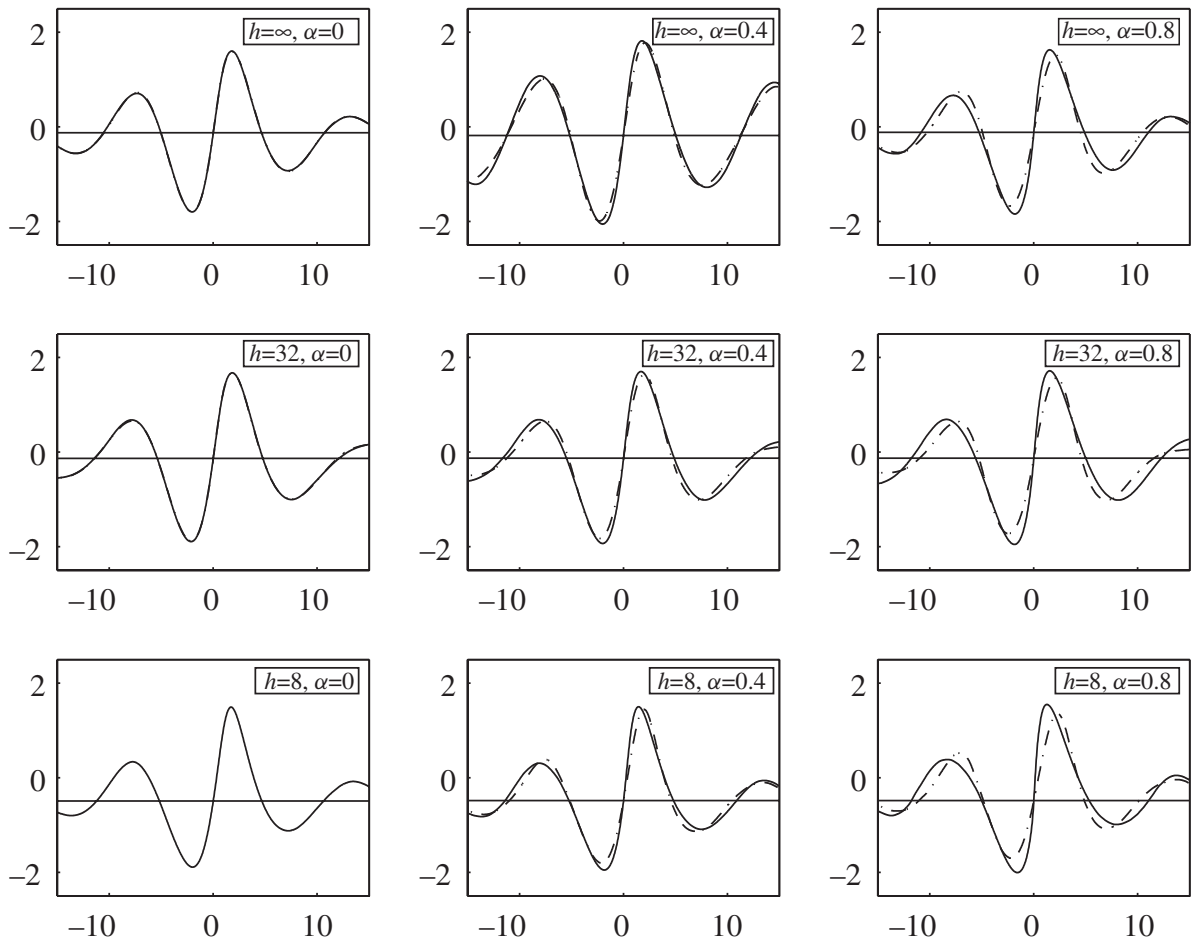

Figure 4: Average shape of upcrossing and downcrossing waves. The solid curves denote the average upcrossing wave shapes centered at upcrossings of the mean level, and the dashed curves denote the reversed downcrossing wave shapes centered at downcrossings. 

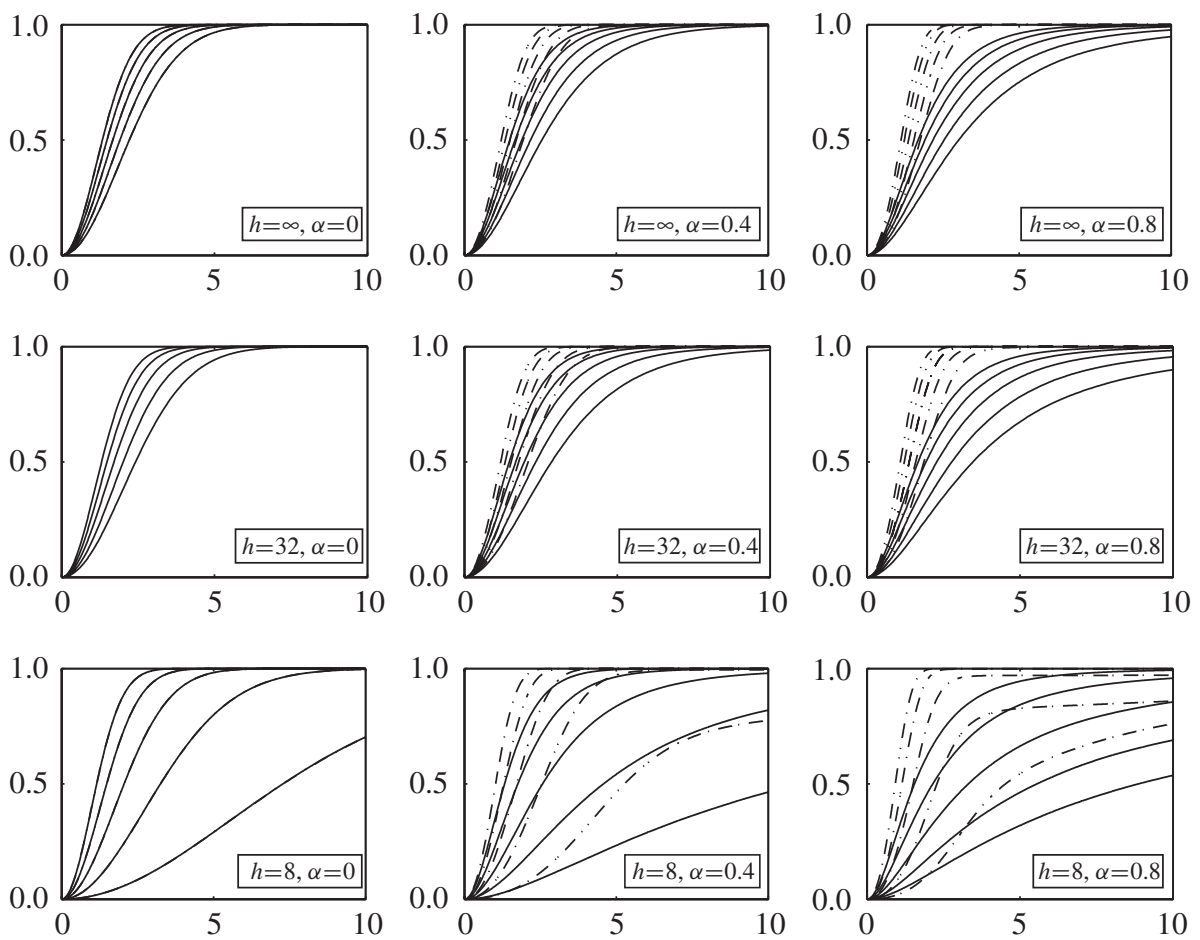

Figure 5: Cumulative distribution functions ( $\mathrm{CDFs}$ ) for time wave slopes (absolute values) at time wave crossings of different levels $v$ : $[-1,0,1,2,3] \times \sigma, 4 \sigma=H_{\mathrm{s}}$. Slope CDFs at upcrossings are denoted by solid lines and slope CDFs at downcrossings are denoted by dash-dotted lines. The largest absolute values correspond to the highest level. The orbital spectrum is the PM spectrum.

5.1.2. Slope in space at time crossings. The analysis makes a combined space-time description possible; case (ST). Figure 6 illustrates the cumulative distribution functions for the slope of the space wave observed when the time wave has an upcrossing or downcrossing of a specified level; all observations refer to a fixed location $x_{0}$. As is natural, the space slope is usually negative when the time wave has an upcrossing, and it is positive at a downcrossing, but there is a small probability of a reverse movement. That means a small wave going in the opposite direction to the main wave direction.

5.1.3. Velocity at time crossings. As a third example of the asymmetry, we have generated the distributions of the horizontal velocity of surface particles at instances of time wave upcrossings and downcrossings; case (VT). The results are shown in Figure 7. Note the regular shift in velocity with increasing level. The zero level is slightly above the mean water level.

\subsection{The forced model}

In this example, the physically motivated linked model (5) will be compared to the forced correlation model (8). For each combination of depth $h$ and $\alpha$-parameter in the linked model, the parameters $\gamma$ and $\delta$ in (8) are chosen to make the variance $\operatorname{var}(X(t, u))$ and covariance $\operatorname{cov}(W(t, u), X(t, u))$ equal in the two models, so the two models have identical bivariate distributions of $(W(t, u), X(t, u))$. 

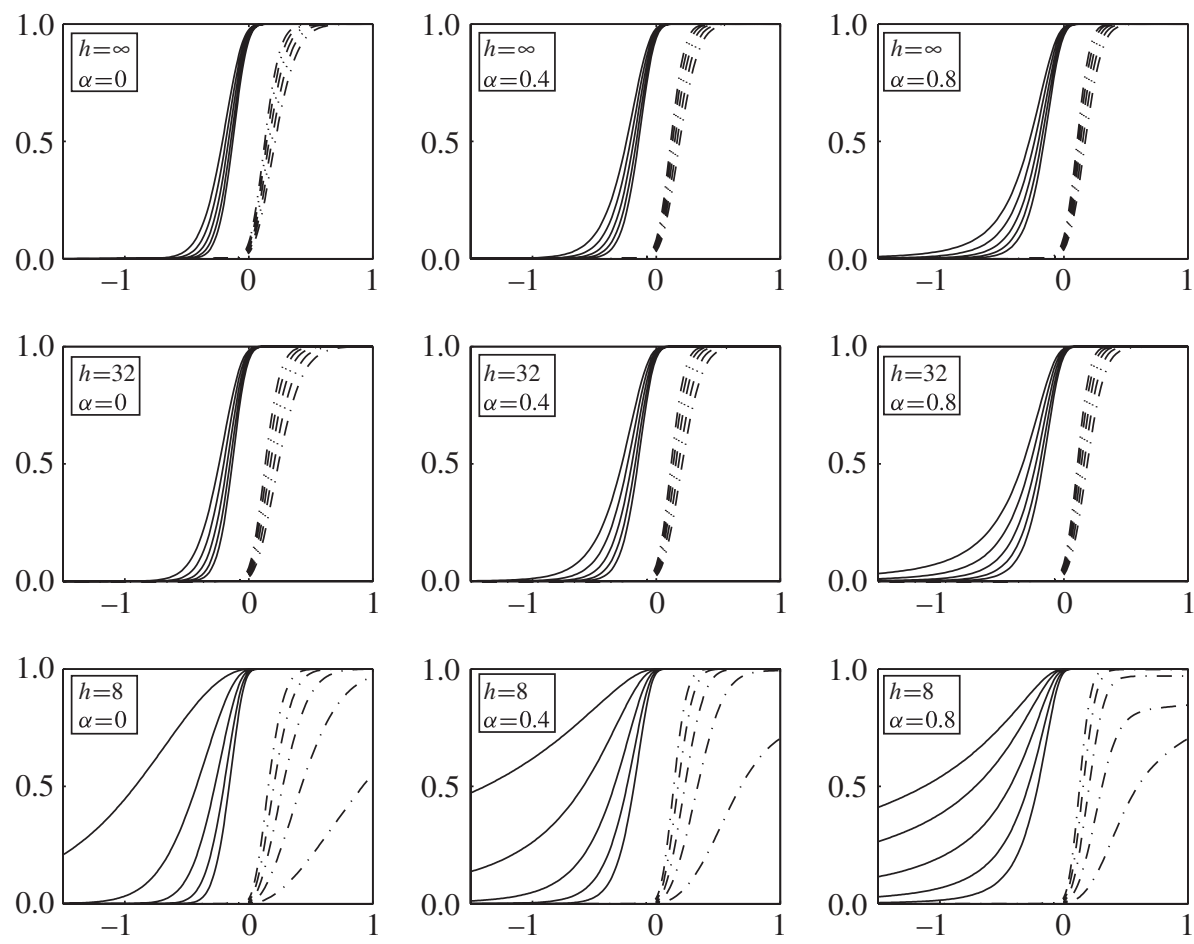

FIGURE 6: CDFs for space wave slopes at time wave crossings of different levels $v$ : $[-1,0,1,2,3] \times$ $\sigma, 4 \sigma=H_{\mathrm{s}}$. Slope CDFs at upcrossings are denoted by solid lines and slope CDFs at downcrossings are denoted by dash-dotted lines. The most extreme values correspond to the highest level. The orbital spectrum is the PM spectrum.

It appears that even if the forced and linked models have the same $(W(t, u), X(t, u))$ distributions, they differ considerably with respect to slope distributions- the forced model giving greater front-back asymmetry. For each of the six asymmetric parameter combinations in Table 1 , the asymmetry in the corresponding distributions in the forced model was analyzed. The relevant asymmetry measures are given in parentheses in Table 1. Figure 8 shows examples of the effect on the average shape of upcrossing and downcrossing waves and TT-slope distributions for the case $h=8 \mathrm{~m}$ and $\alpha=0.4$.

A possible explanation for the increased asymmetry may be the lacking physical realism in the forced model. The inclusion of the $W$-process directly into the $X$-process introduces an unrealistic phase constraint between the two processes. In the free Lagrange process there is a phase shift of 90 degrees, which in the linked model becomes a frequency-dependent phase shift, $\theta(\omega)$, in (7). In the forced model in the example, we normalized the $X$-process to have the same variance and covariance with the $W$-process as in the linked model. This will give a phase shift completely different from that in the linked model. On the other hand, the slope derivative distributions in the two-dimensional space wave depend on three different covariances related to the $X$-process, as shown in [11, Theorem 1], and, hence, it is not possible to calibrate the forced model to nearly the same properties as the linked model. It seems that more detailed studies of water particle velocities in real waves are needed in order to find a good correlation and transfer function structure in the asymmetric first-order Lagrange model; see the discussion in [10]. 

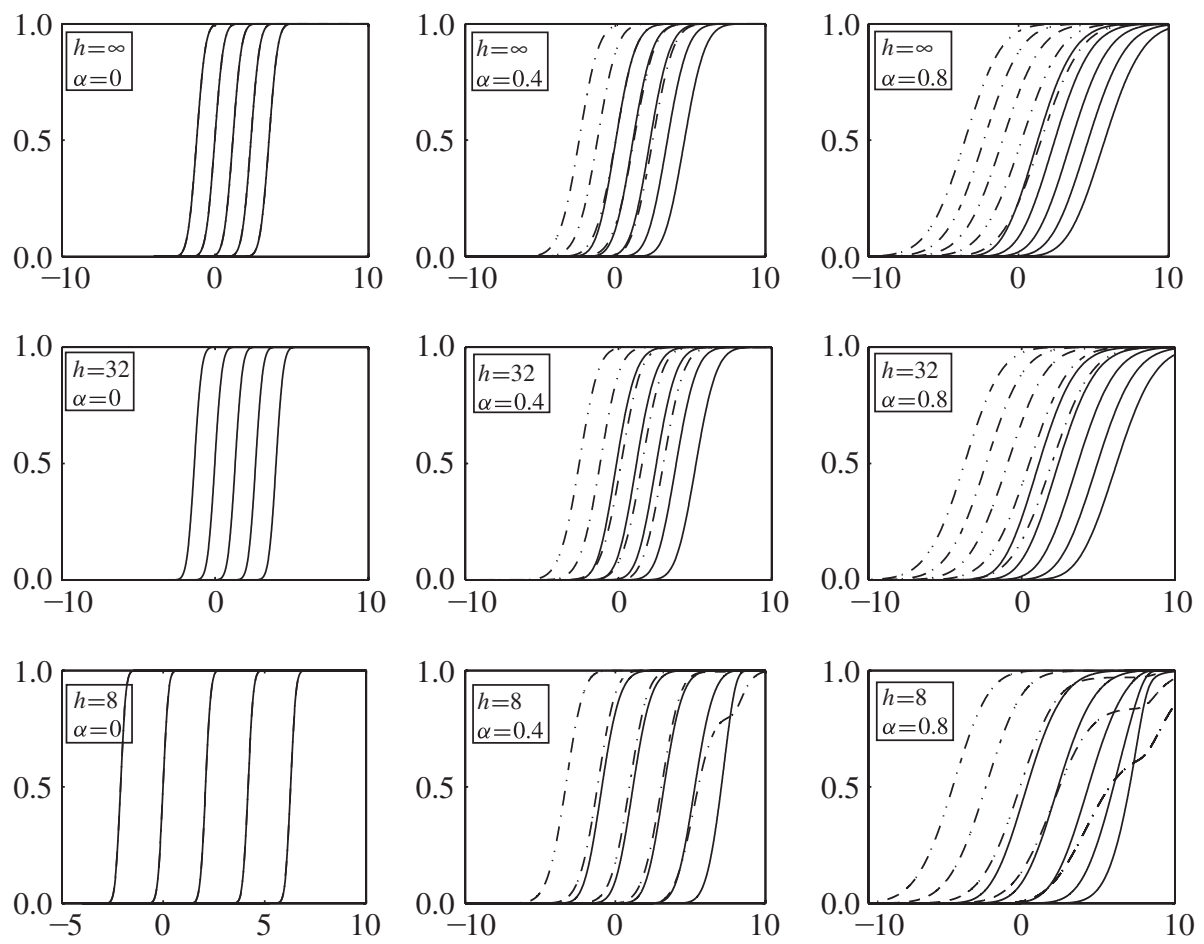

Figure 7: CDFs for the horizontal velocity $(\mathrm{m} / \mathrm{s})$ at time wave crossings of different levels $v$ : $[-1,0,1,2,3] \times \sigma, 4 \sigma=H_{\mathrm{s}}$. Velocity CDFs at upcrossings are denoted by solid lines and velocity $\mathrm{CDFs}$ at downcrossings are denoted by dash-dotted lines. The largest positive velocities correspond to the highest level. The orbital spectrum is the PM spectrum.
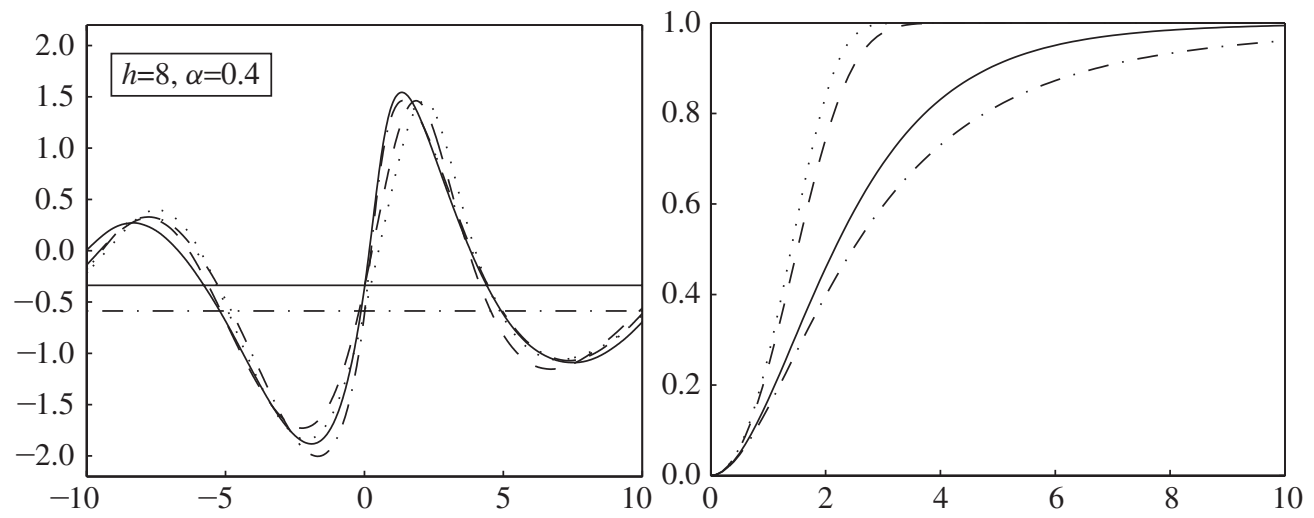

FIGURE 8: Left: mean upcrossing and downcrossing wave shapes in the linked model (solid and dashed curves) and in the forced model (dash-dotted and dotted curves). Right: cumulative distribution functions for time wave slopes (absolute values) at time wave crossings of level $\sigma$ for the linked model (solid and dashed curves) and the forced model (dash-dotted and dotted curves). The diagrams should be compared to the corresponding diagrams in Figures 4 and 5. 


\section{Appendix A. Spectrum and covariances}

The spectrum $S(\omega)$ for the vertical process $W(t, u)$ in the Lagrange model is the orbital spectrum, which is not the same as is observed and estimated from the free water surface. Fouques et al. [5] argued that, for sea states with not so steep waves, the two spectra are very close. As noted in Remark 1, it need not be symmetric; $S(\omega)$ with $\omega>0$ corresponds to waves moving from left to right, $S(\omega)$ with $\omega<0$ corresponds to waves moving in the opposite direction.

The distributions of height and slopes in the Lagrange time and space models depend on the cross-correlations between $W(t, u)=\int \mathrm{e}^{\mathrm{i}(\kappa u-\omega t)} \mathrm{d} \zeta(\omega)$ with orbital spectrum $S(\omega)$ and the filtered process according to (7),

$$
X(t, u)=\int \mathrm{e}^{\mathrm{i}(\kappa u-\omega t)} H(\omega) \mathrm{d} \zeta(\omega),
$$

and their first- and second-order derivatives. These covariances can be found by differentiation of the covariance functions

$$
\begin{aligned}
r^{w w}(t, u) & =\int_{-\infty}^{\infty} \cos (\kappa u-\omega t) S(\omega) \mathrm{d} \omega \\
r^{x x}(t, u) & =\int_{-\infty}^{\infty} \cos (\kappa u-\omega t) \rho(\omega)^{2} S(\omega) \mathrm{d} \omega \\
r^{w x}(t, u) & =\int_{-\infty}^{\infty} \cos (\kappa u-\omega t+\theta(\omega)) \rho(\omega) S(\omega) \mathrm{d} \omega .
\end{aligned}
$$

The slope distributions in Lagrange time waves depend on the joint distribution of the vertical and horizontal processes, and their partial space and time derivatives. Since the two fields are jointly Gaussian, the full six-dimensional distribution is Gaussian, with a covariance matrix built by the covariances, $r_{t t}^{w w}=r_{t t}^{w w}(0,0)=\operatorname{var}\left(W_{t}(0,0)\right), r_{t u}^{w x}=r_{t u}^{w x}(0,0)=$ $\operatorname{cov}\left(W_{t}(0,0), X_{u}(0,0)\right)$, etc.

Using the notation from [1], write

$$
\begin{aligned}
& \boldsymbol{Y}=\boldsymbol{Y}(t, u)=\left(W_{t}(t, u), W_{u}(t, u), X_{t}(t, u), X_{u}(t, u)\right)^{\top}, \\
& \boldsymbol{Z}=\boldsymbol{Z}(t, u)=(W(t, u), X(t, u))^{\top} .
\end{aligned}
$$

Then, the joint distribution of $\left(\boldsymbol{Y}^{\top}, \boldsymbol{Z}^{\top}\right)^{\top}$ is Gaussian with mean $\boldsymbol{\mu}$ and covariance matrix $\boldsymbol{\Sigma}$, partitioned as

$$
\begin{aligned}
& \boldsymbol{\mu}=\left(\begin{array}{llll|ll}
0 & 0 & 0 & 1 \mid 0 & u
\end{array}\right)^{\top}=\left(\boldsymbol{\mu}_{\boldsymbol{Y}}^{\top} \mid \boldsymbol{\mu}_{\boldsymbol{Z}}^{\top}\right)^{\top}, \\
& \boldsymbol{\Sigma}=\left[\begin{array}{cccc:cc}
r_{t t}^{w w} & r_{t u}^{w w} & r_{t t}^{w x} & r_{t u}^{w x} & 0 & r_{t 0}^{w x} \\
r_{u t}^{w w} & r_{u u}^{w w} & r_{u t}^{w x} & r_{u u}^{w x} & 0 & r_{u 0}^{w x} \\
r_{t t}^{x w} & r_{t u}^{x w} & r_{t t}^{x x} & r_{t u}^{x x} & r_{t 0}^{x w} & 0 \\
r_{u t}^{x w} & r_{u u}^{x w} & r_{u t}^{x x} & r_{u u}^{x x} & r_{u 0}^{x w} & 0 \\
\hdashline 0 & 0 & r_{0 t}^{w \bar{x}} & r_{0 u}^{w x} & r_{00}^{w w w} & r_{00}^{w \bar{x}} \\
r_{0 t}^{x w} & r_{0 u}^{x w} & 0 & 0 & r_{00}^{x w} & r_{00}^{x x}
\end{array}\right]=\left[\begin{array}{c:c}
\boldsymbol{\Sigma}_{\mathbf{Y}} & \boldsymbol{\Sigma}_{\mathbf{Y Z}} \\
\hdashline \boldsymbol{\Sigma}_{\mathbf{Z Y}} & \mathbf{\Sigma}_{\mathbf{Z Z}}
\end{array}\right] .
\end{aligned}
$$

Compared to what is the case in the free Lagrange model, the matrix $\boldsymbol{\Sigma}$ gives nontrivial correlation to more of the involved variables; in [1], $\boldsymbol{\Sigma}_{Z Z}$ is diagonal and $\boldsymbol{\Sigma}_{Y Y}$ is block diagonal. 
The conditional distribution of $\boldsymbol{Y}$, given that $\boldsymbol{Z}=(v, 0)^{\top}$, is Gaussian with mean $\boldsymbol{\mu}_{\boldsymbol{Y} \mid \boldsymbol{Z}=(v, 0)^{\top}}$ and covariance matrix $\boldsymbol{\Sigma}_{\boldsymbol{Y} \mid \boldsymbol{Z}}$, given by

$$
\begin{gathered}
\mu_{\boldsymbol{Y} \mid Z=(v, 0)^{\top}=} \mu_{\boldsymbol{Y}}+\boldsymbol{\Sigma}_{\boldsymbol{Y} Z} \boldsymbol{\Sigma}_{Z Z}^{-1}\left(\begin{array}{c}
v \\
-u
\end{array}\right)=A+v B+u C, \quad \text { say } \\
\boldsymbol{\Sigma}_{\boldsymbol{Y} \mid \boldsymbol{Z}}=\boldsymbol{\Sigma}_{\boldsymbol{Y} \boldsymbol{Y}}-\boldsymbol{\Sigma}_{\boldsymbol{Y} \boldsymbol{Z}} \boldsymbol{\Sigma}_{\boldsymbol{Z} Z}^{-1} \boldsymbol{\Sigma}_{\boldsymbol{Z} \boldsymbol{Y}} .
\end{gathered}
$$

Again, in contrast to the free Lagrange case, the matrices $\boldsymbol{A}, \boldsymbol{B}$, and $\boldsymbol{C}$ have no simple structure, in particular, the variable $u$ enters into all the conditional expectations. Furthermore, all the variables in $\boldsymbol{Y}$ are conditionally dependent.

\section{Acknowledgement}

This work was carried out in the framework of the EU project SEAMOCS (contract MRTN CT 2005 019374).

\section{References}

[1] Åberg, S. (2007). Wave intensities and slopes in Lagrangian seas. Adv. Appl. Prob. 39, 1020-1035.

[2] Åberg, S. and Lindgren, G. (2008). Height distribution of stochastic Lagrange ocean waves. Prob. Eng. Mechanics 23, 359-363.

[3] Azaïs, J.-M. And Wschebor, M. (2009). Level Sets and Extrema of Random Processes and Fields. John Wiley, Hoboken.

[4] Elgar, S. (1987). Relationships involving third moments and bispectra of a harmonic process. IEEE Trans. Acoust. Speech Signal Process. 35, 1725-1726.

[5] Fouques, S., Krogstad, H. E. and Myrhaug, D. (2006). A second-order Lagrangian model for irregular ocean waves. Trans. ASME J. Offshore Mechanics Arctic Eng. 128, 177-183.

[6] Gerstner, F. J. (1809). Theorie der Wellen. Ann. Phys. 32, 412-445.

[7] GJøsund, S. H. (2003). A Lagrangian model for irregular waves and wave kinematics. Trans. ASME J. Offshore Mechanics Arctic Eng. 125, 94-102.

[8] LI, W. V. AND WeI, A. (2009). Gaussian integrals involving absolute value functions. In High Dimensional Probability V. The Luminy Volume (Beachwood, Ohio, USA), Institute of Mathematical Statistics, pp. 43-59.

[9] Lindgren, G. (2006). Slepian models for the stochastic shape of individual Lagrange sea waves. Adv. Appl. Prob. 38, 430-450.

[10] Lindgren, G. (2009). Exact asymmetric slope distributions in stochastic Gauss-Lagrange ocean waves. Appl. Ocean Res. 31, 65-73.

[11] Lindgren, G. AND ÅBERG, S. (2009). First order stochastic Lagrange models for front-back asymmetric ocean waves. J. Offshore Mechanics Arctic Eng. 131, 031602-1-031602-8.

[12] Mercadier, C. (2006). Numerical bounds for the distributions of the maxima of some one- and two-parameter Gaussian processes. Adv. Appl. Prob. 38, 149-170.

[13] Miche, M. (1944). Mouvements ondulatoires de la mer en profondeur constante ou décroissante. Forme limit de la houle lors de son déferlement. Application aux digues marines. Ann. Ponts Chassées 1944, 25-78.

[14] Socquet-Juglard, H. et al. (2004). Spatial extremes, shape of large waves, and Lagrangian models. In Proc. Rogue Waves 2004, eds M. Olagnon and M. Prevosto, IFREMER. Available at http://www.ifremer.fr/web-com/ stw2004/rw/fullpapers/krogstad.pdf. 